\title{
A Case Report Of Uterine Leiomyosarcoma
}

This article was published in the following Dove Press journal:

OncoTargets and Therapy

\section{Siyu Cao \\ Yang Liu \\ Xuechai Bai \\ Liang Wang (D)}

Department of Gynecology, The 2nd Affiliated Hospital, Zhejiang University School of Medicine, Hangzhou, People's Republic of China
Correspondence: Liang Wang

Department of Gynecology, The 2nd Affiliated Hospital, Zhejiang University School of Medicine, No. 88 Jiefang Road, Hangzhou, Zhejiang 310009, People's

Republic of China

Tel +86-57I-87783 I28

Email 2196042@zju.edu.cn
Background: Uterine leiomyoma is the most common benign tumor in women. Uterine sarcoma, though with very low incidence, has a high malignant degree and poor prognosis. It has difficulties in preoperative diagnosis, frozen pathological examination and postoperative treatment.

Case report: A 49-year-old woman presented with menstrual disorder. Magnetic resonance imaging showed a huge uterine mass. The patient underwent laparoscopic hysterectomy and part of the uterine tissue looked like fish. Specimens were sent to frozen pathological examination for four times, but none of the results showed malignancy certainly. Considering all abnormalities, we removed the uterine through vagina completely rather than morcellation and did pelvic lymph node biopsy. Postoperative pathological examination revealed uterine leiomyosarcoma and one pelvic lymph node had metastasized.

Conclusion: Uterine sarcoma is difficult to be diagnosed even frozen pathological examination has been performed. Unexpected uterine sarcoma should always be considered, and precautions should be taken if we find anything suspicious. Fortunately, the patient has avoided second operation.

Keywords: uterine leiomyoma, uterine sarcoma, pathological examination, morcellation

\section{Introduction}

Uterine leiomyoma, or uterine fibroid, is a common benign genital tract tumor arising from the myometrium with variable growth rates and can occur at the uterine wall, the lumen, or near the uterine surface. ${ }^{1,2}$ It is characterized in menstrual change, anemia, lower abdominal pain, pain during intercourse and pressure symptoms if the size of tumor is too large. ${ }^{3}$ The factors such as age, obesity, ethnicity, early menarche, using hormonal contraceptive, and family history are associated with the risk of uterine leiomyoma., ${ }^{4,5}$ Uterine sarcomas account for approximately $1 \%$ of all female malignancies gynecologic neoplasm and $3-7 \%$ of all uterine cancers. It was classified into carcinosarcomas, leiomyosarcomas, endometrial stromal sarcomas and undifferentiated sarcomas. Leiomyosarcoma is the most common type of uterine sarcoma and usually occurs in women over 40 years with abnormal vaginal bleeding (56\%), a palpable pelvic mass (54\%) and/or pelvic pain (22\%).

Uterine leiomyoma can undergo various degenerations, such as hyalinization, cystic degeneration, sarcomatoid degeneration and calcification. Approximately 1 in every 800 women believed to have a leiomyoma actually has a sarcoma. ${ }^{6,7}$ Though uterine sarcoma is very rare, it usually causes poor prognosis. ${ }^{8}$ However, preoperative examination, either imaging with ultrasonography or with positron emission tomography scans, is not capable to differentiate benign from malignant smooth muscle masses. 


\section{Case Presentation}

A 49-year-old woman presented to the outpatient service with a 1-year history of menstrual disorder. Laboratory studies showed a luteinizing hormone level of 27.69 international units per liter, a follicle stimulating hormone level of 81.29 international units per liter and a estradiol level of 123.91 pico mole per liter. The determinations of serum sex hormone indicated that the patient was perimenopausal. Transvaginal B-mode ultrasonography and magnetic resonance imaging both showed a huge mixed-sign mass in the uterine cavity (Figure 1). All imaging findings were similar to those of uterine leiomyoma. Due to the huge size of the tumor, laparoscopic hysterectomy was planned to be performed. Diagnostic curettage was performed firstly. The frozen pathological examination found only a small amount of smooth muscle tissue and stratified squamous epithelium. A small piece of myometrial tissue, fish-liking, was taken out though vagina and also sent to frozen pathological examination. It suggested that epithelioid-cell tumor with rich cells and malignant possibility. Considering uterine abnormalities, atrophic ovaries, perimenopausal hormone level, the experience of the chief surgeon and the will of family members, total hysterectomy, bilateral adnexal excision and laparoscopic pelvic lymph node biopsy were performed. In order to avoid the risks linked to morcellation, the big uterus was planned to be removed through the vagina. Oval forceps was used to separate part of uterine contents and take out from vagina. The uterus volume was reduced and could be removed with complete serosa (Figure 2). During this process, part of the uterine content was sent for frozen pathological examination twice. The third examination suggested

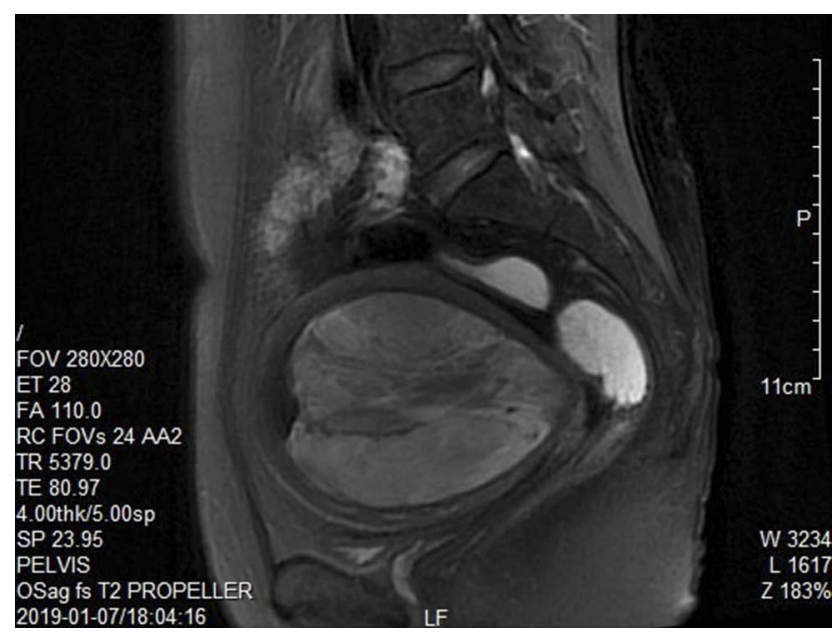

Figure I MRI showed a huge mixed-sign mass in the uterine cavity considering a uterine myoma with degeneration and bleeding.

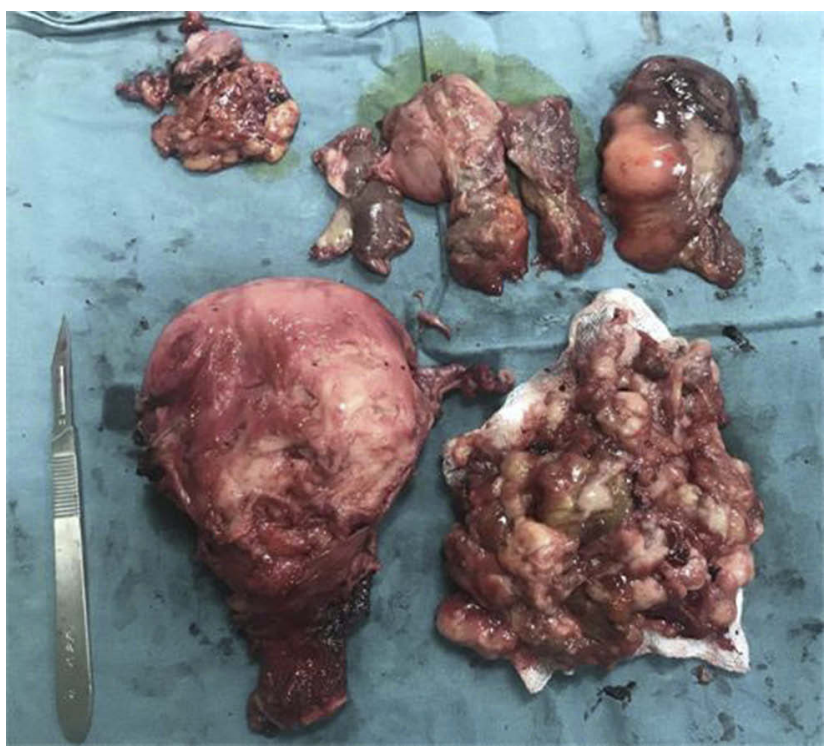

Figure 2 Uterine content was separated from the vagina by oval forceps in order to remove the whole uterus from the vagina completely.

that spindle cell tumors with rich cells and the forth showed uterine myoma with infarct. Nevertheless, postoperative pathological and immunohistochemical analyses of the surgical specimen revealed uterine leiomyosarcoma. Nucleus mitosis and coagulative necrosis were found under magnification $400 \times$ and one pelvic lymph node had metastasized (Figure 3).

\section{Discussion}

With meta-analysis of the literature, the US Food and Drug Administration (FDA) has concluded that the risk of unexpected detection of uterine sarcoma in patients undergoing laparoscopic hysteromyoma surgery was 1/ 352. ${ }^{9}$ Mild-heteromorphism uterine sarcoma is similar to rich-cell uterine leiomyoma in morphology. It is difficult to differentiate them accurately merely by frozen pathological examination. ${ }^{10}$ Immunohistochemical analyses and lymph node biopsy are necessary to reveal the biological behavior of cells and clarify the nature of the tumor. Conditions such as mitotic rate, heterologous elements, necrosis, myometrial invasion, sarcomatous overgrowth and lymphovascular invasion should be taken into account when sarcoma is diagnosed. ${ }^{11,12}$

An integrated medical and surgical approach is usually considered in clinical practice for patients with sarcoma. Risks of dissemination linked to uterine morcellation should be taken into consideration. ${ }^{13}$ Some evidence suggest that survival may be reduced in morcellated sarcoma patients. ${ }^{14}$ In this case, we removed the uterine through 


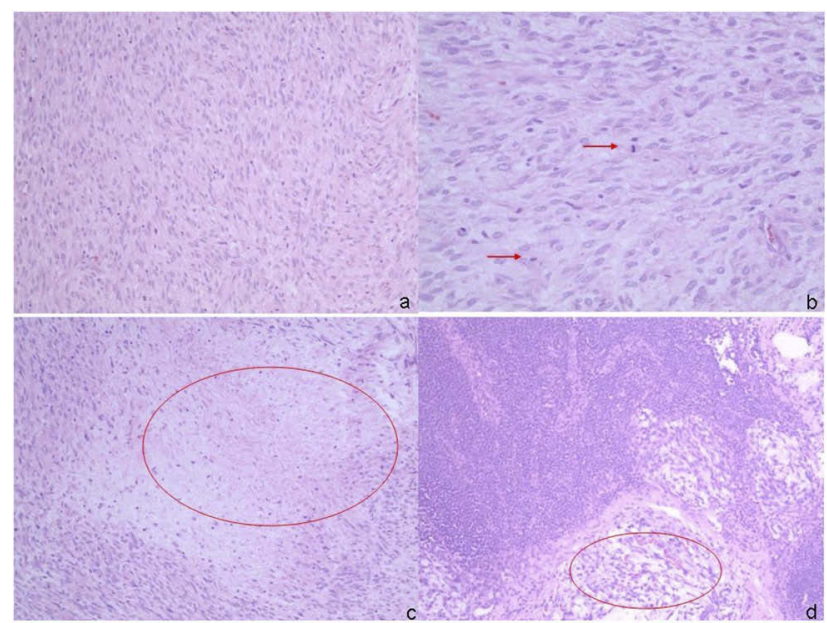

Figure 3 (A) Rich-cell spindle cell tumors with mild heteromorphism (magnification 200x); (B) nucleus mitosis has been marked by arrows (magnification 400x); (C) coagulative necrosis has been circled (magnification 200x); (D) pelvic lymph node metastasis has been circled (magnification 200x).

vagina completely which was extremely difficult but dissemination of tumor cells was effectively prevented.

\section{Conclusion}

Uterine sarcoma is rare and difficult to preoperatively diagnose. Even through clinical features, imageology examination, frozen pathological examination, we cannot obtain an exact diagnosis. In order to find unexpected uterine sarcoma intraoperation, any suspicious details should be taken into account. Dissemination associated with misuse of uterine morcellation and second operation associated with misdiagnosis should be avoided as far as possible.

\section{Ethical Approval}

This study was approved by the ethics committee of the 2nd Affiliated Hospital, Zhejiang University School of Medicine (Hangzhou, People's Republic of China), and was permitted to be published. Written informed consent to have the case details and accompanying images published was obtained from the patient and her husband. All clinical investigations were conducted in accordance with the principles expressed in the Declaration of Helsinki.

\section{Acknowledgments}

This work was supported by grant from the National Natural Science Foundation of China (Grant No. 81472422).

\section{Disclosure}

The authors report no conflicts of interest in this work.

\section{References}

1. Vollenhoven B. Introduction: the epidemiology of uterine leiomyomas. Baillieres Clin Obstet Gynaecol. 1998;12(2):169-176. doi:10.10 16/S0950-3552(98)80059-X

2. Lumsden MA, Hamoodi I, Gupta J, Hickey M. Fibroids: diagnosis and man-agement. BMJ. 2015;351:h4887. doi:10.1136/bmj.h6432

3. Althobaiti FA, Alsaadi KK, Althobaiti AA. A case of hemoperitoneum due to spontaneous bleeding from a uterine leiomyoma. Am J Case Rep. 2019;20:167-170. doi:10.12659/AJCR.914573

4. Okolo S. Incidence, aetiology and epidemiology of uterine fibroids. Best Pract Res Clin Obstet Gynaecol. 2008;22(4):571-588. doi:10.1016/j.bpobgyn.2008.04.002

5. Luesley DM, Kilby MD. Obstetrics \& Gynaecology: An EvidenceBased Text for MRCOG. 3rd ed. CRC Press; 2016.

6. Nappi L, Mele G, Angioni S, Di Spiezio Sardo A, Cicinelli E, Greco P. Uterine leiomyosarcoma: report of three cases and review of the literature. Eur J Gynaecol Oncol. 2014;35(3):328-331.

7. Giuntoli RL, Metzinger DS, DiMarco CS, et al. Retrospective review of 208 patients with leiomyosarcoma of the uterus: prognostic indicators, surgical management, and adjuvant therapy. Gynecol Oncol. 2003;89(3):460-469. doi:10.1016/S0090-8258(03)00137-9

8. Patel SM, Tiwari R, Arora RS, Poddar P, Desai A, Mankad MH. Uterine sarcomas: surgical management, adjuvant therapy and survival outcome. Experience at Gujarat Cancer and Research Institute. Indian $J$ Gynecol Oncol. 2019;17(1). doi:10.1007/s40944-018-0247-0.

9. Liu F, Ling B. Dissemination problems of laparoscopic uterine fibroids morcellation and its prevention. Cancer Research on Prevention and Treatment. 2017;44(9):627-630.

10. Liu FW, Galvan-Turner VB, Pfaendler KS, Longoria TC, Bristow RE. A critical assessment of morcellation and its impacton gynecologic surgery and the limitations of the existing literature. $\mathrm{Am} \mathrm{J}$ Obstet Gynecol. 2015;212:717-724. doi:10.1016/j.ajog.2015.01.012

11. Carroll A, Ramirez PT, Westin SN, et al. Uterine adenosarcoma: an analysis on management, outcomes, and risk factors for recurrence. Gynecol Oncol. 2014. 135: 455-461. doi:10.1016/j.ygyno.2014.10.022

12. Togami S, Kawamura T, Fukuda M, Yanazume S, Kamio M, Kobayashi H. Clinical management of uterine cervical mullerian adenosarcoma: a clinicopathological study of six cases and review of the literature. Taiwan J Obstet Gynecol. 2018;57:479-482. doi:10.1016/j. tjog.2018.04.032

13. Bogani G, Cliby WA, Aletti GD. Impact of morcellation on survival outcomes of patients with unexpected uterine leiomyosarcoma: a systematic review and meta-analysis. Gynecol Oncol. 2015;137 (1):167-172. doi:10.1016/j.ygyno.2014.11.011

14. Bretthauer M, Goderstad JM, Løberg M, et al. Uterine morcellation and survival in uterine sarcomas. Eur J Cancer. 2018;101:62-68. doi:10.1016/j.ejca.2018.06.007 


\section{Publish your work in this journal}

OncoTargets and Therapy is an international, peer-reviewed, open access journal focusing on the pathological basis of all cancers, potential targets for therapy and treatment protocols employed to improve the management of cancer patients. The journal also focuses on the impact of management programs and new therapeutic agents and protocols on patient perspectives such as quality of life, adherence and satisfaction. The manuscript management system is completely online and includes a very quick and fair peer-review system, which is all easy to use. Visit http://www.dovepress.com/ testimonials.php to read real quotes from published authors. 